\title{
Efecto de los alcaliodes del lupinus mutabilis sweet sobre los parásitos gastrointestinal en cuyes
}

\section{Effect of alcaliodes of lupinus mutabilis sweet on gastrointestinal parasites in guinea pigs}

Luis Samuel Eduardo Arias Alemán. ${ }^{1}$, Luis Alejando Ulloa Ramones. ${ }^{2}$, Luis Abdon Rojas Oviedo. ${ }^{3}, \&$ Tamia Elizabeth Noboa Abdo. ${ }^{4}$

\begin{abstract}
DOI: https://doi.org/10.33262/cienciadigital.v3i3.1.692

En el particular ubicado en la parroquia Químiag del cantón Riobamba provincia de Chimborazo, basado en investigaciones realizadas en el ESPOCH se evaluó el aprovechamiento de los alcaloides del Lipinus mutabilis macerado a 0, 72 y 144 holas luego de su cocción un 30 Controlar la presencia de parásitos gastrointestinales para lo que se refiere a 10 animales por tratamiento. La investigación se manejó bajo un diseño completamente al azar y una separación de medios según Duncan al 5\%; para lo que se refiere a los 20 machos destilados de peso promedio de $0.281 \mathrm{~kg}$, los cuales se mantuvieron bajo el estudio de un período de 90 días. Los animales han sido registrados en la presencia de Eimeria sp, Ord Stróngylidea, Paraspidodera uncinata y Trichuris muris. Al analizar los resultados experimentales se determinó que con la utilización del extracto fitoquímico del Lipinus mutabilis sween sin maceración en $1.36 \mathrm{~kg}$ de peso, una ganancia de peso de $1.08 \mathrm{~kg}$, una conversión alimenticia de 5.90 , un peso a la canal de $1.02 \mathrm{~kg}$, el Rendimiento en el canal fue de $74.68 \%$ y un beneficio-costo de 20 centavos determinando por cada dólar invertido se gana 20 centavos. Pudiendo señalar que el suministro del Lipinus mutabilis dulce macerado, controlar la presencia de los parásitos de la fuente de los parámetros productivos.
\end{abstract}

Keywords: Guinea pigs, alkaloids, weight gain, food convertion.

1 Escuela Superior Politécnica de Chimborazo, Sede Morona Santiago, Chimborazo Ecuador, luis.arias@espoc.edu.ec

2 Escuela Superior Politécnica de Chimborazo, Sede Morona Santiago, Chimborazo Ecuador, luis.alejandro86@hotmail.com

3 Escuela Superior Politécnica de Chimborazo, Sede Morona Santiago, Chimborazo Ecuador, taminobo31@gmail.com

4 Escuela Superior Politécnica de Chimborazo, Sede Morona Santiago, Chimborazo Ecuador, luis_ro26@hotmail.com 


\section{Resumen.}

En la particular ubicado la parroquia Químiag del cantón Riobamba provincia de Chimborazo, basado en investigaciones realizadas en la ESPOCH se evaluó la utilización de los alcaloides del Lipinus mutabilis macerado a 0, 72 y 144 holas luego de su cocción un periodo 30 minutos cuya finalidad fue controlar la presencia de parásitos gastrointestinales para lo cual se utilizó 10 animales por tratamiento. La investigación se manejó bajo un diseño completamente al azar y una separación de medias según Duncan al 5\%; para lo cual se utilizó 20 cuyes machos destetados un peso promedio de $0.281 \mathrm{~kg}$, los cuales se mantuvieron bajo el estudio un periodo de 90 días. Los cuyes inicialmente se registraron la presencia de Eimeria sp, Ord Stróngylidea, Paraspidodera uncinata y Trichuris muris. Al analizar los resultados experimentales se determinó que con la utilización del extracto fitoquímico del Lipinus mutabilis sween sin maceración alcanzó $1.36 \mathrm{~kg}$ de peso, una ganancia de peso de $1.08 \mathrm{~kg}$, una conversión alimenticia de 5.90, un peso a la canal de $1.02 \mathrm{~kg}$, el rendimiento a la canal fue de $74.68 \%$ y un beneficio-costo de 20 centavos determinándose que por cada dólar invertido se gana 20 centavos. Pudiendo señalarse que el suministro del Lipinus mutabilis sweet macerado, permitió controlar la presencia de parásitos mencionados inicialmente además de mejorar los parámetros productivos.

Palabras claves: Cuyes, Alcaloides, ganancia de peso, conversión alimenticia.

\section{Introducción.}

Desde nuestros ancestros la utilización del Lipinus mutabilis sweet se ha venido utilizando como un desparasitante debido a que su característica de amargo (alcaloides) hacen que elimine los parásitos en las personas, basados en este conocimiento y en lo manifestado por Cajas, Camacho y Díaz (2008) quienes señalan que el Chocho cocido y macerado permiten controlar la presencia de parásitos en cuyes gracias a que este leguminosa posee propiedades desparasitantes el mismo que ha sido utilizado desde tiempos precolombinos para solucionar problemas de salud en personas y especies domésticas, de esta manera se avizora la posibilidad fundamentar de manera científica el conocimiento ancestral a través del presente estudio y corroborar los trabajos establecidos por las instituciones de desarrollo académico y científico.

Por otro lado el Lupinus mutabilis sweet (Chocho) es una leguminosa de habito erecto, tallos robustos, semileñoso. Alcanza una altura de 0.8-2.0 m. el mismo que se cultiva y adapta a 2.000 y $3.800 \mathrm{~m}$ de altitud cuyo clima es templado y frío; por otro lado se puede mencionar que esta leguminosa es originaria de los Andes de Bolivia, Ecuador y el Perú; se caracteriza por poseer proteínas además de compuestos nitrogenados que dan un sabor especifico a amargo el mismo que se debe someter a un proceso de macerado obligado para poder ser consumido, por otro lado se determina que esta leguminosa tiene interés nutricional en la salud y nutrición del hombre. El alcaloide identificado en esta especie y que actúa como 
amargo se denomina Lupanina (46\%), esparteína (14\%), 4-hidroxilupanina (10\%), isolupanina (3\%), n-metilangustifolina (3\%), 13-hidroxilupanina (1\%), cuyas propiedades son alcalinas, debido a la presencia de nitrógeno básico formando por lo general núcleos heterocíclicos. Estos en forma libre hace que sean insolubles en agua, poco solubles en alcohol y solubles en éter y cloroformo, la mayoría posee oxígeno en su estructura y son sólidos no volátiles, sin embargo algunos no contienen oxígeno como la esparteína, siendo líquida a temperatura ambiente (Bruneton, 1991).

Según estudios preliminares se conoce que los alcaloides son sintetizados en las hojas y en las flores de los lupinus y son transportados a la semilla en desarrollo y al final de las florescencias se encuentran en mayor cantidad (Bruneton, 1991).

De esta manera es posible aprovechar el extracto fitoquímico del chocho por su alto contenido de alcaloides los cuales actúan como desparasitante interno de eespecies domésticas.

\section{Materiales y Métodos}

\section{Localización y Duración del Experimento}

La utilización del Lupinus mutabilis sweet se realizó en una granja particular de la parroquia de Químiag del cantón Riobamba provincia de Chimborazo, la misma que duró 90 días, para lo cual se utilizó como tratamientos diferentes tiempos de maceración ( 0,72 y 144) de chocho cocido a durante 30 minutos cuya efecto de este contenido fue controlar la presencia de parásitos gastrointestinales, este contenido macerado se utilizó en 10 repeticiones por tratamiento, las unidades experimentales se distribuyeron bajo un diseño completamente al azar, y se complementaron sus análisis con la separación de medias según Duncan $(\mathrm{P}<0.05)$.

\section{Procedimiento Experimental}

Adecuación, limpieza y desinfección de las pozas utilizando yodo al $10 \%$. Se utilizaron 30 cuyes machos, de 21 días de edad con un peso promedio de $281 \mathrm{~g}$, los mismos que se distribuyeron en grupos 10 animales por poza y sometidos a un período de 15 días de cuarentena.

La toma de los datos experimentales fueron recogidos y analizados cada 30 días tales como el peso inicial peso final y ganancia de peso, de misma manera se estimó la conversión alimenticia.

Para la determinación de parásitos gastrointestinales se tomaron muestras de heces y llevadas al Laboratorio de Biotecnología para su respectivo análisis coproparasitario. 


\section{Resultados y Discusión}

Inicialmente el peso de los cuyes destetos para el presente trabajo fue de $0.28 \mathrm{~kg}$, determinándose homogeneidad los cuales al comparar con Cajas, Camacho y Díaz (2008) al utilizar chocho macerado, determinan que el peso inicial de los cuyes destetos para la aplicación de chocho macerado a diferentes tiempos fueron de $0.280 \mathrm{~kg}$, siendo similares a los registrados en el presente trabajo experimental (cuadro 1).

A los 90 días se registraron pesos de 1099, 1086 y 1070 g, valores entre los cuales no difieren significativamente $(\mathrm{P}>0.05)$, esto posiblemente se deba a que el manejo que recibieron fueron homogéneos y el tiempo de maceración del chocho fue suficiente, valores que concuerdan con los reportados con cajas los mismos que reportan que el peso de los cuyes a los 90 días en promedio fue $1076 \mathrm{~g}$. Supe (2008) al evaluar plantas desparasitantes como el paico, ajenjo, ruda y marco en el control de parásitos gastrointestinales en cuyes, registro pesos de 0.990 y $1.020 \mathrm{~kg}$, valor similar al de la presente investigación, mientras que Arévalo (2011), señala que el peso luego de los 90 días alcanza peso de $934.95 \mathrm{~g}$.

La ganancia de peso de los cuyes a los 90 días que recibieron extracto de chocho macerado a los 90 días registraron una ganancia de peso de $818,00,805.00$ y 802.00 g respectivamente, valores entre los cuales no difieren significativamente, siendo similares a los registrados por Cajas, Camacho y Díaz (2008) quienes manifiestan que los cuyes ganaron 974 siendo superior a los encontrados en el presente estudio. Supe (2008) encuentro ganancias de pesos de 0.660 y $0.710 \mathrm{~kg}$, Quinatoa (2007) reporta en su investigación ganancias de peso de 0.540 y $0.561 \mathrm{~kg}$, valores inferiores al de la presente investigación.

Cuadro 1. Parámetros productivos de los cuyes bajo el efecto de los alcaloides del Lupinus mutabilis sweet.

\begin{tabular}{lrrrrrr}
\hline & \multicolumn{3}{c}{ Maceración del chocho (horas) } & & \\
\cline { 2 - 4 } \multicolumn{1}{c}{ Variables } & \multicolumn{1}{c}{0} & \multicolumn{1}{c}{72} & 144 & & E.E. & Prob \\
\hline Peso inicial de los animales, g & 281 & 281 & 268 & & \\
Peso a los 90 días, g & $1,099 \mathrm{a}$ & $1,086 \mathrm{a}$ & $1,070 \mathrm{a}$ & 0,030 & 0,056 \\
Ganancia de peso total, g & $818 \mathrm{a}$ & $805 \mathrm{a}$ & $802 \mathrm{a}$ & 0,040 & 0,092 \\
Consumo de materia seca, g & $6018 \mathrm{a}$ & $6855 \mathrm{a}$ & $7007 \mathrm{a}$ & 0,111 & 0,060 \\
Conversión Alimenticia & $7,356 \mathrm{a}$ & $8,51 \mathrm{a}$ & $8.736 \mathrm{a}$ & 0,198 & 0,062 \\
Peso a la canal, g & $822 \mathrm{a}$ & $788 \mathrm{a}$ & $758 \mathrm{a}$ & 0,033 & 0,050 \\
Rendimiento a la canal, \% & $74,79 \mathrm{a}$ & $72,55 \mathrm{a}$ & $70,84 \mathrm{a}$ & 0,861 & 0,058 \\
\hline
\end{tabular}

Letras iguales no difieren estadísticamente $(\mathrm{P}<0.05)$ según Duncan.

E.E.: Error estándar para el periodo de maceración del chocho

El consumo de materia seca de los cuyes a los 90 días de la investigación fue de 6018, 6855 y $7007 \mathrm{~g}$, valores que estadísticamente no fueron significativas $(\mathrm{P}>0.05)$ siendo ligeramente inferiores a los registrados por Cajas, Camacho y Díaz (2008) puesto que registran un 
consumo de $7886 \mathrm{~g}$ en el periodo de engorda, lo que significa que el consumo de aliento de los cuyes es superior al registrado en el presente trabajo. Supe (2008) registró consumos de materia seca de 4.630 y $4.890 \mathrm{~kg}$, valores inferiores al de la presente investigación.

La conversión alimenticia en un periodo de 90 días luego del destete fuer de 7.35, 8.56 y 8.73 , valores entre los cuales no difieren significativamente $(\mathrm{P}>0,05)$ al comparar entre las medias de los tratamientos, los cuales al comparar con Cajas, Camacho y Díaz (2008), Yucailla (2013) reportan conversiones de 6.32 siendo más eficiente que los alcanzados en el presente estudio, esto posiblemente se deba a la digestibilidad de los alimentos y a otros factores intrínsecos de los cuyes incluso al grupo genético que permite eficiencias de transformación de alimento en ganancia de peso de los animales. Supe (2008), reporta conversiones de 6.69 y 7.13, Quinotoa (2007) alcanzó 8.12 y 8.25 conversiones de alimento, siendo eficiencias alimenticias similares a presente investigación.

Los cuyes bajo el efecto del chocho macerado a los 90 días del destete alcanzaron pesos a la canal de 822,788 y $758 \mathrm{~g}$, valores entre los cuales no registraron diferencias significativas ( $P>0,05)$, aunque al comparar con Cajas, Camacho y Díaz (2008) los pesos a la canal de los cuyes fueron de $921 \mathrm{~g}$, siendo superior en promedio, esto puede deberse a muchos factores en estudio, principalmente a la genética de los animales y consigo a la capacidad de transformar el alimento en ganancia de peso. Zarate, A. (2006), al utilizar diferentes tipos de alimentación alcanzó pesos a la canal entre 0.610 y $0.880 \mathrm{~kg}$. Casa (2008) reporta pesos a la canal de $0.810 \mathrm{~kg}$, valores similares a los registrados en el presente estudio.

El rendimiento a la canal de los cuyes sometidos al agua de chocho macerado fue de 74.79, 72.55 y $70.84 \%$, valores entre los cuales no difieren significativamente ( $P>0.05)$, los cuales al comparar con Cajas, Camacho y Díaz (2008) reportan que el rendimiento a la canal fueron de $73.32 \%$ en promedio, el mismo que está dentro del rango establecido en el presente trabajo de investigación. Zarate, A. (2006), alcanzó rendimientos a la canal de 65.13 y 73.58 $\%$ los cuales están dentro de los alcanzados en el presente trabajo de investigación, mientras que Garcés (2003) y Mullo (2009), señalan que los rendimientos a la canal están entre 69,71 y 79,66 \% valores extremos que difieren de los encontrados en el presente estudio.

En el cuadro 2, la presencia de Eimeria sp al inicio de la investigación en cuyes bajo el efecto del extracto de chocho macerado a las 0,72 y 144 horas fueron 6704.16, 9379.16 y 4350.25 de Ooquistes por gramo de heces, y a los 90 días de la aplicaicon de los respectivos tratamientos se redujo a $30.83,35.00$ y 5.16 Ooquistes por gramo de heces respectivamente. Supe (2008) al aplicar diferentes plantas con efecto desparasitante para el control de parásitos gastrointestinales en cavia porcellus reporta que la presencia de Eimeria $s p$ encontró de 50 a 450 Ooquistes por gramo siendo inferiores a los alcanzados en el presente estudio aunque Cajas, Camacho y Díaz (2008) reportan cargas parasitarias semejantes a las encontradas en el presente estudio.

La presencia de parásitos Ord. Strongylidea en cuyes machos al inicio de la investigación registraron 200.00, 253.33 y 188.33 Huevos por gramo de heces respectivamente, los cuales 
al suministrar el extracto de chocho macerado se redujo en su totalidad a los 90 días, por lo que se puede señalar que estos alcaloides fueron eficientes para controlar este tipo de parásitos, los cuales corroboran por lo señalado por Cajas, Camacho y Díaz (2008).

Cuadro 2. Efecto del extracto de chocho macerado y cocido en la presencia de parásitos en los cuyes machos en la fase de engorde.

\begin{tabular}{lrrrrr}
\hline & \multicolumn{3}{c}{ Tiempo de Maceración } & & E.E. \\
\cline { 2 - 4 } \multicolumn{1}{c}{ Variables } & \multicolumn{1}{c}{0} & \multicolumn{1}{c}{72} & \multicolumn{1}{c}{144} & Media & $\mathrm{t}(0,05)$ \\
\hline Eimeria sp, Inicial, OPG & 6704,16 & 9379,16 & 4350,25 & 6811,19 & 2847,31 \\
Eimeria sp a los 90 días, OPG & 30,83 & 35,00 & 5,16 & 16,66 & 12,47 \\
Ord. Strongylidea inicial, HPG & 200,00 & 253,33 & 188,33 & 163,88 & 75,63 \\
Ord. Strongylidea a los 90 días, OPG & 0,00 & 0,00 & 0,00 & 0,00 & 0,00 \\
Paraspidodera uncinata inicial, HPG & 510,40 & 650,00 & 589,55 & 549,99 & 136,12 \\
Paraspidodera uncinata a los 90 días, & & & & & \\
HPG & 5,16 & 30,16 & 8,16 & 12,58 & 16,30 \\
Trichuris muris inicial, HPG & 70,00 & 42,50 & 61,66 & 34,62 & 22,20 \\
Trichuris muris a los 90 días, HPG & 0,00 & 0,00 & 0,00 & 0,00 & 0,00 \\
\hline
\end{tabular}

OPG: Ooquistes por gramo de heces

HPG: huevos por gramo de heces

\section{Paraspidodera uncinata}

La carga parasitaria de Paraspidodera uncinata en cuyes destetos fue 510.40, 650.00 y 589.99 huevos por gramo de heces, los mismos que al someter al extracto de chocho macerado a los 90 días redujo a 5.16, 30.16 y 8.16 Huevos por gramo de heces, resultados similares a los registrados por Cajas, Camacho y Díaz (2008) los cuales reportan una carga parasitaria de 4.16 e incluso a los 120 días se reporta una eliminación total de este parasito.

Finalmente la presencia de Trichuris muris en cuyes destetos fueron de 70, 42.50 y 61.66 Huevos por gramo de heces, los mismos que a los 90 días se redujo en su totalidad, comportándose de la misma manera como demuestra Cajas, Camacho y Díaz (2008), de esta manera se demuestra que este producto del chocho macerado es eficaz para controlar la presencia de Trichuris muris en cuyes. Supe (2008) señala que el Truchuris sp, no se elimina no se eliminan fácilmente al utilizar extractos de plantas con alcaloides sin embargo el extracto de chocho cocinado y macerado macerado resulta eficiente.

\section{Conclusiones}

- Los cuyes evaluados inicialmente presentaron infestación por: Eimeria sp, Ord Stróngylidea, Paraspidodera uncinata y Trichuris muris, como parásitos de 
asentamiento gastrointestinal, los cuales fueron controlados con la aplicación del extracto de chocho macerado.

- Aunque no se determinó diferencias significativas, la utilización del chocho macerado este producto no influyó en los parámetros productivos.

Por lo tanto se recomienda:

- Utilizar el extracto proveniente del Lupinus mutabilis sweet como desparasitante interno puesto que controla la proliferación de parásitos como la Eimeria sp, Ord Stróngylidea, Paraspidodera uncinata y Trichuris muris.

\section{Referencias Bibliográficas}

Arévalo, M. (2011). Efecto de la utilización del ajo macerado (allium sativum) en el control de yersinia pseudotuberculosis y escherichia coli en cuyes, etapa crecimiento engorde. Tesis de grado. Facultad de Ciencias Pecuarias. ESPOCH. Riobamba Ecuador.

Bruneton, J. (1991). Elementos de Fotoquímica y Farmacología.- Trad. por. Ángel Villar del Fresno. se. Zaragoza, España. Edit. Acriba. pp.12-14.

Cajas, A. Camacho, C. Díaz, H. (2008) Efecto de la utilización del chocho (Lupinus mutabilis sweet) como antiparasirario gastrointestinal en cuyes bajo diferentes tiempos de maceración y cocción. Tesis de grado. Facultad de Ciencias Pecuarias. ESPOCH. Riobamba - Ecuador.

Casa, C (2008) Efecto de la utilización de forraje verde hidropónico de avena, cebada, maíz y trigo en la alimentación de cuyes. Tesis de grado. Facultad de Ciencias Pecuarias. ESPOCH. Riobamba - Ecuador.

Caicedo, G. (2004). Manual de Investigación y Evaluación Fotoquímica de diferentes plantas medicinales. sn. Pasto, Colombia. se. p 23

Garcés, S. (2003). Efecto del uso de la cuyinaza más melaza en el balanceado en la alimentación de cuyes. Tesis de Grado. Facultad de Ciencias Pecuarias, ESPOCH. Riobamba, Ecuador. pp. 21 - 73.

Mullo, (2009). Aplicación del promotor natural de crecimiento (Sel - plex) en la alimentación de cuyes mejorados (Cavia porcellus) en la etapa de crecimiento engorde y gestación - lactancia. Tesis de Grado. ESPOCH, FCP, EIZ

Quinatoa, S (2007). Evaluación de diferentes niveles de harina de retama más melaza en la elaboración de bloques nutricionales para la alimentación de cuyes. Tesis de grado. Facultad de Ciencias Pecuarias. ESPOCH. Riobamba - Ecuador.

Supe, C (2008). Utilización de plantas desparasitantes tradicionales: paico, ajenjo, ruda y marco en El control de parásitos gastrointestinales en cuyes. Tesis de Grado. EIZ FCP - ESPOCH. Riobamba Ecuador.

Yucailla, V. (2013). Utilización de agua de chocho, guarango, alcachofa y marco, como desparasitantes naturales, para el control de piojos en cuyes en la granja agroturistica totorillas. Tesis de Grado. EIZ - FCP - ESPOCH. Riobamba Ecuador. 
Zarate, A. (2006). Utilización de cuatro tipos de alimentación y su efecto en crecimiento y engorde de cuyes machos mejorados. Tesis de Grado. EIZ - FCP - ESPOCH. Riobamba Ecuador.

PARA CITAR EL ARTÍCULO INDEXADO.

rias Alemán, L. S. E., Ulloa Ramones, L., Rojas Oviedo, L., \& Noboa Abdo, T. (2019). Efecto de los alcaliodes del lupinus mutabilis sweet sobre los parásitos gastrointestinal en cuyes. Ciencia Digital, 3(3.1), 221-228. https://doi.org/10.33262/cienciadigital.v3i3.1.692

\section{Ligital}

El artículo que se publica es de exclusiva responsabilidad de los autores y no necesariamente reflejan el pensamiento de la Revista Ciencia Digital.

El artículo queda en propiedad de la revista y, por tanto, su publicación parcial y/o total en otro medio tiene que ser autorizado por el director de la Revista Ciencia Digital.
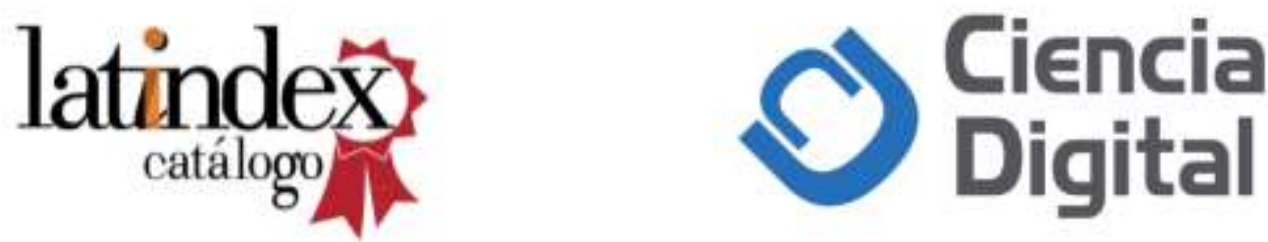